\title{
Algoritmos de Monitoreo para Baterías Recargables
}

\section{Algorithms for Monitoring Rechargeable Batteries}

Presentación: 06/10/2020

\section{Doctorando:}

\section{Facundo Quiñones}

Facultad Regional La Plata, Universidad Tecnológica Nacional - Argentina - facundo.quinones@fain.uncoma.edu.ar

\section{Director/a:}

\section{Ruben H. Milocco}

\section{Co-director/a:}

\section{Silvia G. Real}

\section{Resumen}

El monitoreo inteligente de baterías es un componente fundamental de los sistemas de almacenamiento basados en estos dispositivos ya que garantiza el correcto y seguro uso de la energía. Dentro de las principales tareas que se realizan en estos sistemas se encuentra la de estimar y predecir, a partir de las mediciones de corriente, voltaje y temperatura, variables e indicadores de las baterías como el estado de carga, el envejecimiento y la energía disponible.

En este trabajo se presentan algoritmos que permiten conocer y predecir algunas de estas variables a partir de un modelo basado en dos procesos electroquímicos que dan lugar a la conversión de energía química en eléctrica en una batería. En particular, se trabajó sobre el cálculo de tiempo remanente y la estimación del estado de carga y de una variable adicional que modela el efecto de rate-capacity. Los algoritmos propuestos fueron probados sobre un banco formado por cuatro baterías comerciales de iones de litio. Los resultados obtenidos indican que la estrategia propuesta permite predecir el tiempo remanente con un error medio de 4,2 minutos en descargas de hasta 1 hora.

Palabras clave: Modelo electroquímico, batería recargable, estado de carga, tiempo remanente.

\begin{abstract}
The battery management system is a fundamental part of the battery-based storage system due to allow use the energy in a safety way. Ones of the main tasks of this system includes the estimation and prediction of the state of charge, the aging and the remaining energy all from voltage, current and temperature measurement.

In this work, we present algorithms to perform some of these estimations using an electrochemical model based on two processes that allow the battery converts the chemical energy intothe electrical energy. In particular, the prediction of the remaining time and the estimation of the state of charge and other additionally variable that models the rate-capacity effect were carried on. The proposed algorithms were tested in a battery pack formed by four commercial Li-ion batteries connected electrically in series. The results shows that the averaged error in the prediction of the remaining time was 4.2 minutes considering discharges lesser and equal to 1 hour of duration.
\end{abstract}

Keywords: Electrochemical Model, rechargeable battery, state of charge, remaining time.

\section{Introducción}

En la actualidad cada vez son más las aplicaciones que utilizan baterías para abastecer la energía necesaria para su funcionamiento. En dispositivos portátiles de uso cotidiano, en vehículos y hasta en parques eólicos y solares se utilizan sistemas de almacenamiento basados en ellas. Cuando la capacidad de almacenamiento no puede ser provista por una batería individual, se 
utilizan bancos formados por decenas y hasta centenas de ellas. Básicamente, en un banco las baterías se conectan eléctricamente en serie y/o en paralelo para conseguir una determinada capacidad de almacenamiento de energía. Estas conexiones pueden combinarse de diferentes maneras, aunque una de las topologías más utilizada, sobre todo en vehículos eléctricos, es conectar baterías en paralelo formando módulos y luego conectar varios de estos módulos en serie formando lo que se conoce como un pack de baterías, (Shen \& Gao, 2019, págs. 2-33).

El diseño de estos sistemas de almacenamiento se completa incluyendo un conjunto hardware-software llamado BMS (por sus siglas en inglés correspondientes a Battery Management System) que se encarga de tres tareas básicas con el fin de garantizar una gestión segura y eficiente de la carga almacenada, (Waag, Fleischer, \& Uwe Sauer, 2014). Estas tareas pueden resumirse en: mantener a cada una de las baterías trabajando dentro de límites seguros de tensión, corriente y temperatura; estimar y calcular indicadores y parámetros de las baterías y ecualizar las tensiones de estas. Dentro de los indicadores estimados por el BMS los más utilizados en la mayoría de las aplicaciones son el estado de carga o SoC (State of Charge), el estado de salud o SoH (State of Health) y la energía y/o potencia disponible. Este último parámetro está estrechamente relacionado con el tiempo remanente, siendo este el tiempo en el que la batería (o el banco) está disponible para entregar una determinada corriente.

En este trabajo se desarrolla una estrategia que permite predecir el tiempo remanente (TR) en bancos de baterías utilizando para tal fin un modelo electroquímico que describe el comportamiento dinámico de una batería. El algoritmo desarrollado utiliza un observador de estados que permite además estimar el estado de carga de todo el conjunto.

\section{Modelo electroquímico de una batería}

Una batería recargable es un sistema electroquímico formado por dos electrodos insertados en un medio electrolítico donde por medio de reacciones de reducción-oxidación (redox) es posible entregar y almacenar cargas en el interior de la misma. En la región de contacto entre electrodo (conductor de electrones) y el electrolito (conductor de iones) se genera una interfase donde se produce la transferencia de cargas. Si bien en ambos electrodos ocurren estas reacciones redox, el proceso global es controlado por la interfase en la que el consumo de reactivos se produce a mayor velocidad. Este electrodo se llama limitante y es el que define la diferencia de potencial en bornes de la batería. Suponiendo sin pérdida de generalidad que este es el cátodo, la corriente que entrega la batería, $I$, puede describirse en función de la concentración superficial de reactivos en este electrodo, $X$, y del potencial de este utilizando la ecuación de Butler-Volmer, (Bockris, Reddy, \& Gamboa-Aldeco, 2000, págs. 1052-1055), como sigue:

$$
I=k^{r}(1-X) e^{b(1-\beta) E c}-k^{o} e^{-b \beta E c}
$$

Donde Ec es el potencial del cátodo y la concentración superficial $X \in\left[\begin{array}{ll}0 & 1\end{array}\right]$ está normalizada con respecto a la máxima concentración que puede ser acumulada en la interfase. Las constantes $k^{o}$ y $k^{r}$ dependen de la velocidad de la reacción de oxidación y reducción y de la concentración máxima de reactivos, $\beta \in\left[\begin{array}{ll}0 & 1\end{array}\right]$ es el factor de simetría de la reacción, $\mathrm{b}=\mathrm{F} / \mathrm{RT}$ donde $\mathrm{R}$ es la constante universal de los gases, $\mathrm{F}$ es la constante de Faraday y $\mathrm{T}$ es la temperatura.

Por otro lado, la diferencia de potencial en bornes, $E$, de la batería está dado por $E=E c-E a-I R_{i}$, donde $E a$ es el potencial del ánodo (electrodo no limitante) y $R_{i}$ representa todas las pérdidas óhmicas debido al transporte y transferencia de cargas en el interior de la batería. Reemplazando $E c$ de esta en la ecuación (1) se tiene que:

$$
I=K^{r}(1-X) e^{b(1-\beta)(E-I R)}-K^{o} e^{-b \beta(E-I R)}
$$

Donde $K^{r}=k^{r} e^{\left(E a-E_{q}\right)}$ y $K^{o}=k^{r} e^{\left(E a-E_{q}\right)}$. La ecuación (2) puede factorizarse de una manera conveniente que permite encontrar una expresión para la diferencia de potencial en bornes. Para ello primero se despeja el cociente $X /(1-X)$ resultando en la ecuación (3) y luego el lado derecho de esta se factoriza convenientemente como una función exponencial resultando en la ecuación (4):

$$
\begin{gathered}
\frac{X}{1-X}=\frac{K^{o} e^{b \beta(E-I R)}-I}{K^{r} e^{-b \beta(E-I R)}+I} \\
\frac{X}{1-X}=\frac{K^{o}}{K^{r}} e^{b \beta\left(E-I R_{e q}\right)}
\end{gathered}
$$


De esta manera se obtiene la siguiente relación entre la corriente, la diferencia de potencial y la concentración de reactivos del electrodo limitante:

$$
E(t)=f(X(t))-I(t) R_{e q}
$$

En esta descomposición, la función $f(X(t))$ modela la dependencia de la tensión (o diferencia de potencial) con la concentración de reactivos, mientras que los efectos que no dependen de esta concentración están agrupados en la $R_{e q}$. Las expresiones de éstas dos funciones están dadas por:

$$
\begin{gathered}
R_{e q}=R_{i}+\frac{1}{2 b I} \ln \left(\frac{1-I e^{-b \beta(E-I R)} / K^{o}}{1+I e^{-b \beta(E-I R)} / K^{o}}\right) \\
f(X)=K_{a}+K_{b} \ln \left(\frac{X}{1-X}\right)
\end{gathered}
$$

La función $f(X(t))$ representa a la fuerza electromotriz (EMF) de la batería, siendo esta la energía (por unidad de carga eléctrica) que impulsa a la corriente a través del circuito externo.

La segunda parte del modelo está relacionada con la dinámica de difusión de los reactivos en el electrodo limitante que da lugar a la concentración X. Este proceso de transporte hacia la interfase puede modelarse utilizando las leyes de Fick y es posible demostrar que la relación dinámica entre la corriente $I$ y $X$ puede modelarse con sistemas lineales e invariantes en el tiempo. Más aún la relación entre estas dos variables puede escribirse como un integrador puro seguido de un filtro lineal pasa altos infinitodimensional de ganancia unitaria en estado estacionario que llamaremos $G(s)$, (Milocco, Castro, \& Thomas, 2014, págs. 609620). El integrador puro, de ganancia $-1 / Q_{\text {max }}$, modela el $\mathrm{SoC}$ y el filtro $G(s)$ modela la dinámica de la batería relacionada con el efecto de rate-capacity (RCE). El RCE es el efecto que origina que la batería no se comporte como un acumulador puro y se manifiesta en la dependencia de la capacidad de la batería (cantidad de carga eléctrica entregada) con la amplitud de la corriente de descarga. Básicamente, debido a que el transporte de sustancia reactiva es un proceso difusional, la concentración $X$ en la intefase decrecerá, durante la descarga, más rápido que la concentración de reactivos en el seno de la disolución. Es por esto que grandes corrientes de descarga producen un rápido agotamiento de los reactivos disponibles en la interfase imposibilitando la transferencia de cargas aun cuando existe sustancia reactiva en el interior de la batería. De igual manera, el RCE está relacionado con el efecto de recuperación que se manifiesta por un aumento de la tensión al anular la corriente de descarga. Una representación en diagramas de bloques del modelo completo se muestra en la Figura 1.

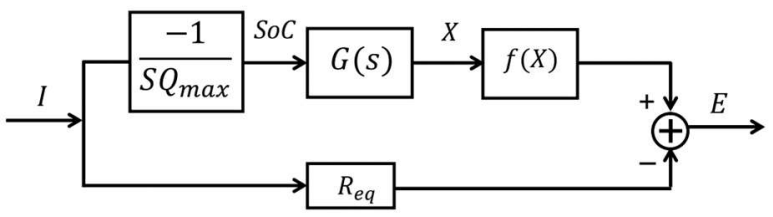

Figura 1: Diagrama en bloques del modelo electroquímico de una batería recargable.

\section{Predicción de tiempo remanente}

El tiempo remanente, $\Delta_{t}$, de una batería recargable es el período de tiempo en que la batería puede descargarse a corriente constante hasta que su tensión en bornes alcance un valor mínimo preestablecido que llamaremos $E_{m}$ (para detalles ver (Quiñones, Milocco, \& Real, 2018, págs. 256-263)). Llamando I a la amplitud de la corriente constante de descarga, utilizando la ecuación (5) y suponiendo que se conoce $R_{e q}$ se sabe que:

$$
E_{m}=f\left(X\left(\Delta_{t}\right)\right)-I R_{e q}
$$

Por otro lado, para conocer la evolución temporal de las variables del modelo de la Figura 1 es necesario definir una estructura para el subsistema $G(s)$. Para tal fin se propone utilizar una función de transferencia de primer orden, siendo esta una aproximación muy utilizada en diversos trabajos, ya que a la hora de llevar a cabo una implementación de un BMS se busca utilizar reducidos parámetros. La función de transferencia de primer orden $\left(G^{1}(s)\right.$ ) puede expresarse con la ecuación (9) y la respuesta de las dos variables del sistema para tiempos $t>0$ ante una corriente constante quedan dadas por las ecuaciones (10) - (11). 


$$
G^{1}(s)=\frac{a s+1}{p s+1} ; \quad 0<p<a
$$

$$
\operatorname{SoC}(t)=\operatorname{SoC}(0)-t I / Q_{\max }
$$

$$
X(t)=\operatorname{SoC}(0)\left(1-e^{-t / p}\right)+X(0) e^{-t / p}+\left((p-a)\left(1-e^{-\frac{t}{p}}\right)-t\right) I / Q_{\max }
$$

Donde $a$ y $p$ son los parámetros del sistema que modela la dinámica del RCE y $\operatorname{SoC}(0)$ y $X(0)$ son las condiciones iniciales de estas dos variables (es decir el valor que tenían previo a aplicar la corriente al sistema). Evaluando la ecuación (11) en $t=\Delta_{t}$ es posible conocer $X\left(\Delta_{t}\right)$ e igualar esta expresión a la que resulta al despejar este mismo factor de (8), de esta manera se llega a una expresión del tipo:

$$
\begin{gathered}
\Delta_{t}+\theta_{1}+\theta_{2} e^{-\Delta_{t} / p}=0 \\
\theta_{1}=Q_{\text {max }}\left(X\left(\Delta_{t}\right)-\operatorname{SoC}(0)\right) / I-(p-a) \\
\theta_{2}=Q_{\max }(\operatorname{SoC}(0)-X(0)) / I-(p-a)
\end{gathered}
$$

Note que las constantes $\theta_{1}$ y $\theta_{2}$ se conocen al momento de comenzar la descarga. Finalmente, la ecuación (12) se resuelve utilizando la función de Lambert $W[\cdot]$. Para esto, primero se multiplica toda la ecuación por el factor $(1 / p) e^{\left(\Delta_{t}+\theta_{1}\right) / p}$ para llegar a una ecuación de la forma de (15).

$$
w e^{w}=\alpha
$$

Donde, $\alpha=-\left(\theta_{2} / p\right) e^{\theta_{1} / p}$ y $w=\left(\Delta_{t}+\theta_{1}\right) / p$. La solución a esta ecuación es $w=W[\alpha]$. Finalmente, el tiempo remanente queda expresado por:

$$
\Delta_{t}=w p-\theta_{1}
$$

\section{Predicción de tiempo remanente en bancos de baterías}

En esta sección se extiende el cálculo del TR al caso de un banco de baterías formado por la conexión de baterías en serie. En este sentido, se define el tiempo remanente de un banco como el período tiempo en que el banco puede descargarse a corriente constante hasta que al menos una de sus baterías alcance el valor de $E_{m}$ en sus bornes. Las hipótesis que se considerarán en relación al banco de baterías son las siguientes: i) todas las baterías del pack son controladas con un único BMS que se encarga de monitorear a todo el conjunto y tiene acceso a las mediciones de todas las tensiones de las baterías individuales, ii) todas las baterías son del mismo tipo y tiene el mismo envejecimiento, por lo tanto el valor de los parámetros $\left(a, p, Q, R_{e q}, f(\cdot)\right)$ del modelo que las representa serán similares. Puede verse que una manera de calcular el TR de todo el conjunto, es utilizar un modelo como el utilizado en la sección anterior, para cada una de la baterías, luego predecir el TR de cada una de ellas y finalmente seleccionar el mínimo de entre todos, ya que el TR del conjunto lo define la batería que primero alcance el valor de $E_{m}$ en sus bornes. Sin embargo, la cantidad de parámetros y las cuentas necesarias del algoritmo de esta estrategia aumenta conforme lo hace el número de baterías del banco. Con el fin de reducir la cantidad de parámetros se propone en este trabajo utilizar un único modelo para modelar a todas las baterías de banco. El modelo se utilizará en conjunto con un observador estados (un filtro de kalman) que permite estimar el valor $S o C$ y $X$ para predecir luego el TR del conjunto. Para esto la propuesta es corregir las estimaciones calculadas por el observador utilizando el valor de tensión de la batería que esté más próxima al valor $E_{m}$. Esta estrategia es útil en términos del TR ya que en todo momento el observador se conduce a estimar las variables de estado de la batería con menor tensión siendo esta la candidata a definir el TR del conjunto. El esquema de trabajo se resume en la Figura 2. 


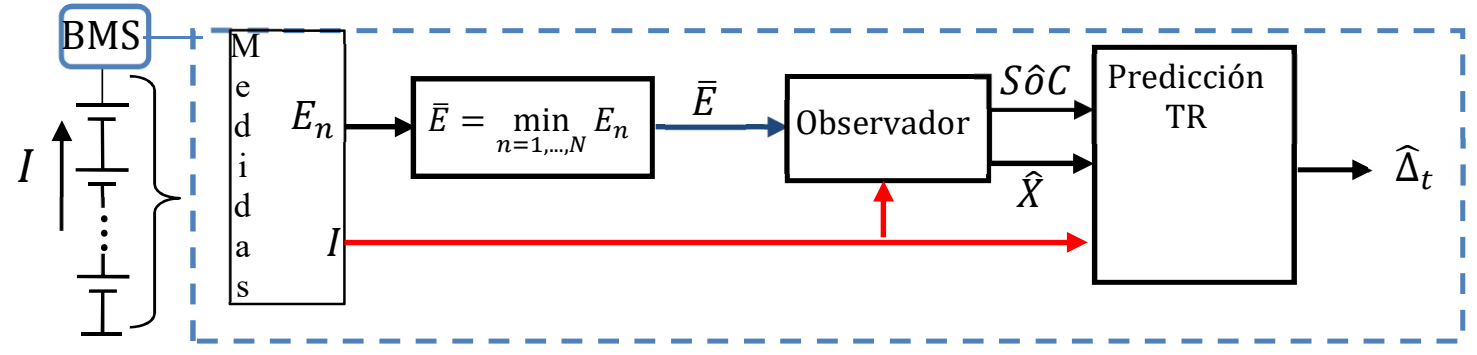

Figura 2: Esquema de trabajo propuesto para predecir el tiempo remanen de un banco de baterías.

El algoritmo del observador es el siguiente (ver, (Song \& Grizzle, 1992, págs. 3365-3369) para detalles):

1. $\mathrm{k}=1$. Adquirir: $I(k)$ y $\bar{E}(k)$.

2. $K(k)=\frac{Q^{-}(k) C^{T}(k)}{C(k) Q^{-}(k) C^{T}(k)+r_{2}}$

3. $\hat{z}(k)=\hat{z}^{-}(k)+K(k)\left(\bar{E}(k)-C(k) \hat{z}^{-}(k)+I(k) R_{e q}\right)$

4. $Q^{-1}(k)=\left(Q^{-}(k)\right)^{-1}+\frac{C^{T}(k) C(k)}{r_{2}}$

5. $\quad \hat{z}^{-}(k+1)=A\left(h_{s}\right) \hat{z}(k)+B\left(h_{s}\right) I(k)$

6. $Q^{-}(k+1)=A\left(h_{s}\right) Q(k) A^{T}\left(h_{s}\right)+R_{1} R_{1}^{T}$

7. $k=k+1$. Ir al paso 2 .

$$
\begin{aligned}
& A\left(h_{s}\right)=\left[\begin{array}{cc}
1 & 0 \\
1-e^{-t / p} & e^{-t / p}
\end{array}\right] \\
& B\left(h_{s}\right)=\left[\begin{array}{cc}
-t & (p-a)\left(1-e^{-\frac{t}{p}}\right)-t
\end{array}\right] \frac{1}{Q}
\end{aligned}
$$

El algoritmo comienza con un valor inicial de $Q^{-}(0)$ y los valores conocidos de la varianza de ruido en la salida $\left(r_{2}\right)$ y matriz de covarianza de ruido en los estados $R_{1}$. Se ha utilizado una notación matricial para describir a las ecuaciones (10) - (11) descritas por la matriz $A\left(h_{s}\right)$ y el vector $B\left(h_{s}\right)$. Además, el vector z contiene los dos estados del modelo y el símbolo $\hat{z}$ corresponde a valores estimados. Las matrices $Q$ y $Q^{-}$son las matrices de covarianza del error de estimación de los estados a priori y a posteriori y $h_{s}$ es el periodo de muestreo de manera que $t=k h_{s}$; siendo $k$ un entero. Una vez que se obtienen las estimaciones de $\hat{z}$, se obtiene la estimación $\widehat{\Delta}_{t}(k)$ con la ecuación (16) utilizando $S \hat{o} C(k)$ y $\widehat{X}(k)$ en las expresiones (13) y (14).

\section{Resultados experimentales}

Los resultados mostrados en esta sección corresponden a un banco de baterías conectadas en serie formado por 4 baterías comerciales de iones de litio cuyo ánodo es de grafito y el cátodo está compuesto por Nickel-Manganeso y óxido de Cobalto (Liion NMC). Primero se identificaron los parámetros de los modelos de las baterías utilizando un procedimiento que consiste en descargar a la batería con una corriente periódica formada por pulsos de descargas seguida de un período de relajación. El procedimiento comienza con la batería totalmente cargada y los pulsos son aplicados hasta que se alcanza el valor de tensión mínima dada por el fabricante. En el caso de las baterías utilizadas este valor es 3,4V. Cada pulso de descarga decrementa una cantidad de carga de tal manera que al sumar todas las contribuciones a lo largo del ensayo permite estimar la capacidad $Q_{\max }$. Suponiendo que se inicia de una condición de $\mathrm{SoC}=1$, se obtiene el $\mathrm{SoC}$ a lo largo del ensayo utilizando la ecuación (10). Luego, la función $f(\cdot)$ se obtiene considerando distintos pares de puntos (SoC, OCV), siendo el OCV el valor de la tensión en bornes al final de cada período de relajación. Este período debe ser lo suficientemente grande como para que el transitorio del RCE se extinga. Finalmente, los parámetros restantes se obtienen minimizando la suma del cuadrado del error entre la tensión medida y la modelada con la ecuación (8) a partir del modelo (10) - (11). En la Figura 3 a) se muestra medición de tensión y la modelada por el modelo con los parámetros identificados obtenidos sobre una de las cuatro baterías. En la Figura 3 b) se muestra la función $f(\cdot)$ obtenida a partir de interpolar los puntos obtenidos con el procedimiento descrito.

(a)



Figura 3 a): Azul: tensión medida en el ensayo de identificación. Azul: tensión modelada. (b)

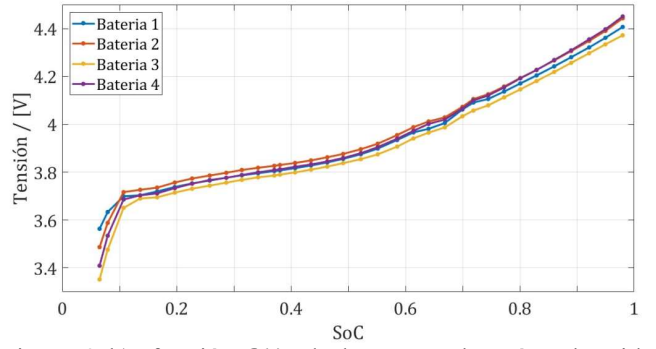

Figura 3 b): función $f(\cdot)$ de las cuatro baterías obtenidas con el ensayo propuesto para la identificación 
Los valores representativos de los parámetros de las cuatro baterías obtenidos fueron: $Q_{\max }=1.3 \mathrm{Ah} ; a=0,7 \mathrm{~h} ; p=$ $0,46 h ; R_{e q}=0,23 \Omega$. Estos valores corresponden al valor promedio entre los obtenidos para cada una de ellas.

Una vez identificados los modelos, se realizaron ensayos para para evaluar la predicción del TR con la estrategia propuesta. Para esto se utilizaron ensayos que consistieron en aplicar al banco distintos perfiles de corriente formados por pulsos continuos de carga y descarga. La corriente se mantuvo constante durante la descarga hasta que la tensión de alguna de sus baterías alcanzo el valor de $E_{m}=3,4 \mathrm{~V}$. Seguido a esto el conjunto fue cargado hasta que la tensión de alguna de sus baterías alcanzó el valor de $3,4 \mathrm{~V}$. Las amplitudes de las corrientes de carga y descarga fueron elegidas aleatoriamente en el intervalo $[0,7 A, 2,6 A]$. De todos los ensayos, se obtuvo un conjunto de 32 descargas que se utilizaron para evaluar la predicción del TR. Las predicciones realizadas fueron comparadas a posteriori con el TR real obtenido a partir de contabilizar el tiempo que le tomo a al menos una de las baterías alcanzar el valor límite admisible $E_{m}$ en sus bornes. Por cada descarga se obtuvo el costo $J$ dado por la ecuación (17) que representa la raíz cuadrada del error medio cuadrático.

$$
J=\sqrt{\sum_{k=1}^{N} \frac{\left(\Delta_{t}(k)-\widehat{\Delta}_{t}(k)\right)^{2}}{N}}
$$

En esta ecuación, $N$ es la duración de cada descarga considerada. En la Figura 4 a) se muestra este valor en función de la duración total de la descarga y el valor medio (en línea punteada) de este costo obtenido en las 32 descargas consideradas. Estas líneas indican que en promedio se cometió un error de 4,2 minutos. En cuanto a los resultados del observador, en la Figura 4 b) se muestra la medida de la tensión de las cuatro baterías del pack y la estimada por el filtro de kalman (en líneas punteadas) a lo largo de uno de los ensayos utilizados para predecir el TR. Puede verse que siempre el observador se encarga de estimar el menor valor de entre todas las tensiones.

(a)

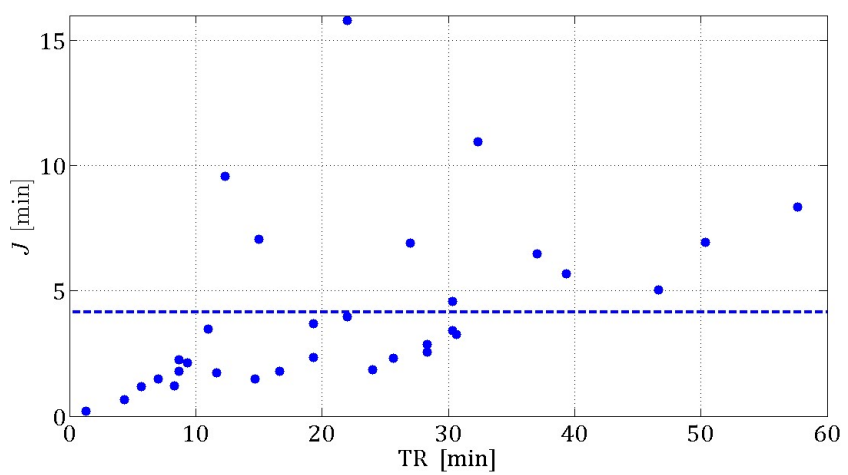

Figura 4 a): Valores del costo obtenidos en la predicción del tiempo remanente, en función de la duración de la descarga. (b)



Figura 4 b): Registro de las tensiones de las baterías y estimación obtenida con el filtro de kalman en uno de los ensayos de carga/descarga utilizado para predecir el tiempo remanente.

\section{Conclusiones}

Se presentó un modelo electroquímico que es capaz de representar el comportamiento dinámico de una batería recargable. Este modelo se utilizó para predecir el tiempo remanente de un banco de baterías formado por cuatro baterías en serie. Utilizando 30 ensayos de carga/descarga sobre baterías de iones de Litio comerciales se evaluó el algoritmo propuesto. El desempeño en la predicción se evaluó utilizando un costo que es la raíz cuadrada del error medio cuadrático, obteniendo en promedio un error de 4,2 minutos en descargas de hasta 1 hora. El algoritmo propuesto utiliza un filtro de kalman como observador de estado que se encarga en todo momento de estimar los estados de la batería cuya tensión es la menor de entre todas las del banco.

\section{Referencias}

Bockris, J., Reddy, A., \& Gamboa-Aldeco, M. (2000). Modern Electrochemistry, Fundaments of Electrodics, 2nd edition. New York: Kluwer Academic Publishers.

Milocco, R., Castro, B., \& Thomas, J. (2014). Generic dynamic model of rechargeable batteries. J. of Power Sources, 609-620.

Quiñones, F., Milocco, R., \& Real, S. (2018). Remaining discharge-time prediction for batteries using the lambert function. J. of Power Sources, 256-263.

Shen, M., \& Gao, Q. (2019). A review on battery management system from the modeling efforts to its multiapplication and integration. Int. J. of Energy Research, 2 - 33. 3365-3369.

Song, Y., \& Grizzle, J. (1992). The extended kalman filter as a local asymptotic observer for nonlinear discrete-time systemst. ACC/FM12,

Waag, W., Fleischer, C., \& Uwe Sauer, D. (2014). Critical review of the methods for monitoring of lithium-ion batteries in electric and hybrid vehicles. J. of Power Sources, 321-339. 